\title{
CONCORDANCIA PLURAL EN FORMAS VERBALES INFINITIVAS DEL ESPAÑOL DE COSTA RICA
}

\author{
Jorge Antonio Leoni de León
}

\section{(9) $\mathbb{P Q \Theta \Theta}$}

Doi: https://doi.org/10.15517/rfl.v46iExt..43936

URL: https://revistas.ucr.ac.cr/index.php/filyling/index 



\title{
CONCORDANCIA PLURAL EN FORMAS VERBALES INFINITIVAS DEL ESPAÑOL DE COSTA RICA
}

\author{
PLURAL AGREEMENT IN INFINITIVE VERBS OF \\ COSTA RICAN SPANISH
}

\author{
Jorge Antonio Leoni de León
}

\begin{abstract}
RESUMEN
En el español de Costa Rica, al igual que en otras variedades, las secuencias clíticas, con un pronombre final en objeto indirecto singular, pueden aceptar un plural referido anafóricamente a un sujeto; es el caso, por ejemplo, de "tienen que ponersen vivos", donde la $\{-n\}$ concuerda con el sujeto de la oración principal. Esto produce el efecto de una concordancia de infinitivo, algo que, en principio, excluye la morfología de las formas infinitivas en español estándar. En este artículo, abogamos por un abordaje global de este tipo de plural, al ponerlo en relación con otras estructuras similares.

Palabras clave: morfosintaxis; pronombres clíticos; concordancia; infinitivo; pluralidad.
\end{abstract}

\begin{abstract}
In Costa Rican Spanish, as in other varieties, clitic sequences, with a final pronoun in singular indirect object, can accept a plural mark which refers anaphorically to a subject; this is the case, for example, "tienen que ponersen vivos", where the $\{-n\}$ agrees with the subject of the main sentence. This produces the effect of infinitive agreement, something that, in principle, is excluded by the morphology of non-finite forms in standard Spanish. In this article, we advocate a global approach to this type of plural, by putting it in relation to other similar structures.

Keywords: morphosyntax; clitic pronouns; agreement; infinitive; plurality.
\end{abstract}

Dr. Jorge Antonio Leoni de León. Profesor de la Escuela de Filología, Lingüística y Literatura. Universidad de Costa Rica. Costa Rica.

Correo electrónico: antonio.leoni@ucr.ac.cr 


\section{Introducción ${ }^{1}$}

Los pronombres átonos o clíticos son un fenómeno general del español (Pérez, 2000), ampliamente conocido, que constituye una de sus características más relevantes. Los pronombres clíticos dependen de una unidad anfitriona para su realización fonética; en nuestra lengua, los verbos cumplen esa función. Según la posición en la que se realizan los clíticos con respecto al verbo (en adelante $\mathrm{V}$ ), podemos clasificar sus secuencias como enclíticas (1b) o proclíticas (1d).

(1) a. Ella quiso escribir esa novela.

b. Ella quiso escribirla.

c. Ella escribió esa novela.

d. Ella la escribió.

(2) a. Ella quiso escribir [esa novela $]_{i}$.

b. Ella quiso escribirla $t_{\mathrm{i}}$.

c. Ella la $\mathrm{i}$ quiso escribir $t_{\mathrm{i}}$.

Los pronombres clíticos en (1a) se interpretan con respecto a la estructura argumental del verbo (2), así (1a) y (1b), al igual que (1c) y (1d), son equivalentes. Además, estos pronombres tienden a formar secuencias, como en (3b) y en (3c), equivalentes a (3a), según un patrón secuencial definido que, contrario a otras lenguas, como el francés, no varía ni en la enclisis ni en la proclisis (Bastida, 1975).

(3) a. Ella le pudo entregar la novela a la editorial.

b. Ella se la pudo entregar.

c. Ella pudo entregársela.

(4) a. Ella le $\mathrm{j}_{\mathrm{j}}$ pudo entregar [la novela $]_{\mathrm{i}}$ [a la editorial $]_{\mathrm{j}}$.

b. Ella se ${ }_{i} l_{\mathrm{j}}$ pudo entregar $t_{\mathrm{i}} t_{\mathrm{j}}$.

c. Ella pudo entregárse $\mathrm{la}_{\mathrm{j}} t_{\mathrm{i}} t_{\mathrm{j}}$.

Los ejemplos en (3) ilustran la capacidad de agrupamiento y secuencialidad de los pronombres clíticos, así como la influencia del rasgo [ \pm persona] del verbo para su realización con respecto a su categoría anfitriona, siendo las formas enclíticas determinadas por $\mathrm{V}_{\text {[-persona] }}$, es decir, por el infinitivo y el gerundio (en español la incorporación de clíticos al participio es un fenómeno rarísimo); los pronombres enclíticos se realizan inmediatamente después del morfema infinitivo. Los clíticos también se posponen en las formas de imperativo ("dáselo, cómpralo"), pero se anteponen en las formas negativas ("no me lo des", "no lo compres"). El rasgo de persona también es relevante en el orden de los pronombres en las secuencias clíticas (Perlmutter, 1970). El ejemplo (4) representa la compleja estructura de relaciones entre los argumentos del verbo y la capacidad de los pronombres clíticos de actuar como unidades morfológicas que sustituyen sintagmas argumentales nominales o preposicionales ("le" y "se" para "a la editorial" y "la" para "la novela"), de objeto directo o indirecto.

1 Este artículo es uno de los resultados del proyecto Análisis de mecanismos de coherencia sintáctica y semántica del español (745-B7097) del Instituto de Investigaciones Lingüísticas, el cual ha contado con el apoyo de la Vicerrectoría de Investigación de la Universidad de Costa Rica. 
En este artículo, abordaremos la incorporación de los pronombres clíticos en el infinitivo, particularmente, en la construcción subordinada infinitiva con fuerte prevalencia del rasgo [ \pm plural]. El infinitivo, por definición, carece de morfemas de concordancia en el español estándar. Consecuentemente, las subordinadas infinitivas no concuerdan ni con el verbo ni con el sujeto de la oración principal, tal y como lo ilustran los ejemplos (5a) y (5c). No obstante, en el español coloquial de Costa Rica, no es raro escuchar oraciones infinitivas con secuencias pronominales enclíticas en las que el morfema plural del verbo, $\{-n\}$, se manifiesta en posición final de palabra, inmediatamente después de la secuencia clítica; este es el caso de "ponersen" y "salirsen" en (5b) y (5d), donde se produce una concordancia con el sujeto de la oración principal (es decir, ellos).

(5) a. Tienen que ponerse vivos.

b. Tienen que ponersen vivos.

c. Ellos quieren salirse.

d. Ellos quieren salirsen.

Este fenómeno, conocido como transposición de plural, infinitivo personal, infinitivo flexionado, concordancia infinitiva o, incluso, infinitivo conjugado, consiste, en español, en la adición de un morfema de número en la posición final de una oración infinitiva subordinada, tema que abordaremos en este artículo.

La concordancia infinitiva no es infrecuente en el español coloquial; sus particularidades, divididas según las categorías y las funciones involucradas, como veremos más adelante, invitan a analizar cada una por separado. Sin embargo, la pregunta que nos planteamos es si no sería más adecuado abordar este fenómeno desde una perspectiva más amplia. Así, en este artículo, pretendemos relacionar la concordancia infinitiva en español con otros fenómenos similares que también involucran morfemas de plural, los cuales no se limitan a una relación anafórica con un sujeto, sino que también se pueden extender, de diversas maneras, con el objeto directo o indirecto.

La concordancia infinitiva ha sido abordada desde diversas perspectivas, Harris y Halle (2005) se focalizaron en el imperativo, mientras Manzini y Savoia (2011) extendieron este análisis al romance y el albanés. Mare (2018) describe la aparición de lo que denomina “-n enclítica" en varios contextos sintácticos del español americano, anotando que también ha sido atestiguado históricamente en el español peninsular en distintos períodos. Pato y de Benito (2017) se refieren a la trasposición de plural en las secuencia clítica "nos lo" convertida en el equivalente "no los" en formas imperativas e infinitivas del español americano; por nuestra parte, no encontramos rastro de este tipo de secuencia en el español de Costa Rica. Sánchez Corrales (1987), con consideración de estudios anteriores, se refiere a la presencia de este fenómeno en Costa Rica, desde el punto de vista del cambio lingüístico como forma de resolver la ambigüedad referencial, pero también como resultado de las razones internas del sistema.

En la sección 2 de este artículo describiremos la concordancia infinitiva en el español de Costa Rica. Luego, en la sección 3, apuntaremos argumentos a favor de una visión global del plural en posición final de secuencias clíticas. En la sección 4, resumimos las conclusiones.

\section{La concordancia de infinitivo en el español de Costa Rica}

El plural del verbo en la tercera persona se forma en español con el morfema $\{-n\}$, 
para los tres temas del infinitivo: $\{-\operatorname{ar}\},\{-e r\}$ e $\{-i r\}$. En consecuencia, tenemos "ama, aman" para "amar"; "come, comen", para "comer"; "sale, salen" para "salir"; este morfema también se manifiesta en los verbos irregulares, como "pone, ponen" para "poner", "va, van" para "ir" y "es, son" para "ser". Siendo que el infinitivo en español estándar carece de rasgos de concordancia, no hay ningún morfema que exprese, en el infinitivo, rasgos como persona o número (6a) y (6c). Sin embargo, en el español coloquial, es posible encontrar el morfema plural del verbo $\{-\mathrm{n}\}$, al final de las secuencias enclíticas de tercera persona, tal y como se ilustra en (6b) y (6d).

(6) a. Ellos tienen que irse.

b. Ellos tienen que irsen.

c. Dejen de ponerse en payasadas.

d. Dejen de ponersen en payasadas.

Este plural no pertenece a la secuencia clítica, sino que resulta de una relación anafórica con un elemento externo, incluso ajeno a sus referentes, que, en (6b) y (6d), identificamos como el sujeto de la oración principal. Por esta razón, hablamos de plural exclítico. Este plural tiene mucha vitalidad en Costa Rica como lo demuestran los ejemplos (7)-(13), donde la secuencia enclítica, incluyendo el plural exclítico, está marcada en negrita; el referente original está subrayado. En las frases b de cada ejemplo, las cadenas de referencia de (7)-(13) están indicados con paréntesis angulares (“<...>”), los círculos negros “•” marcan el límite de los clíticos y el plural en posición final de palabra.

(7) a. Yo y cada uno de nosotros pagamos las dietas de los diputados. Los elegimos creyendo en que eran capaces para tomar las acciones y decisiones para sacar este país adelante, no para ponersen a inventar estupideces por su incompetencia en lo que realmente necesita este país.

b. $\operatorname{Los}_{<\text {los diputados> }}$ elegimos creyendo en que eran capaces para tomar las acciones y decisiones para sacar este país adelante, no para poner $\bullet \mathbf{s e}^{\bullet} \mathbf{n}_{<\operatorname{los} \text { diputados }>}$ a inventar estupideces por su incompetencia en lo que realmente necesita este país.

Tipo: Comentario.

Fuente: Mora Hernández (2015).

(8) a. La Administración tiene conocimiento del problema de este puente, pero quienes deben ponersen de acuerdo son los vecinos, porque no podemos hacer si no hay unión de opiniones.

b. La Administración tiene conocimiento del problema de este puente, pero quienes deben poner $\bullet \mathbf{s e}^{\bullet} \boldsymbol{n}_{<\text {quienes }}$ de acuerdo son los vecinos, porque no podemos hacer si no hay unión de opiniones.

Tipo: Acta.

Fuente: Consejo Municipal de Santa Ana (2014).

(9) a. En cuanto al alegato de los recurrentes de que no hay suficientes asientos en la Sala de sesiones del Concejo Municipal, el amparo se desestima, toda vez que, la autoridad recurrida bajo juramento informó que se trata de una situación temporal, la cual será superada cuando los enseres que fueron guardados ahí con ocasión al terremoto 
ocurrido el pasado 8 de enero, se reubiquen, volviendo a colocarsen los asientos.

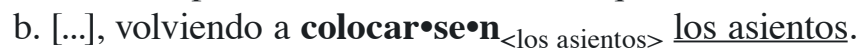

Tipo: Sentencia.

Fuente: Sala Constitucional de la Corte Suprema de Justicia (2009).

(10) a. Durante todo el trámite del juego el Real Madrid fue superior a la Roma, con un fútbol de mucha posesión pero sin tener la contundencia necesaria, situación que provocó que la primera anotación llegara tarde, a punto de irsen al descanso.

b. [...], a punto de ir•se• $\mathbf{n}_{<\text {ellos (sujeto desinencial)> }}$ al descanso.

Tipo: Noticia.

Fuente: Chaves (2018).

(11) a. Para el tercer cuarto los locales mantendrían la ventaja a favor de 61 a 52, pero sería hasta el último cuarto que los poetas mediante buena defensa y ataque lograron empatar el marcador 68 a 68 y en los último 3 minutos irsen arriba en el marcador hasta finalizar 70 a 79 a favor de los ramonense lo que los convierte en los campeones invictos del torneo, logrando su tercer titulo nacional en el Baloncesto.

b. [...] y en los último 3 minutos ir॰se $\bullet n_{<\text {los poetas }>}$ arriba en el marcador hasta finalizar 70 a 79 [...]

Tipo: Noticia.

Fuente: Comunicados Vive 506 CR (2017).

(12) a. Porque tampoco puedo permitir como lo iban a hacer la semana pasada, que mandan un correo invitando a la gente a irsen a ver sin solicitarme autorización para hacer una actividad de estas.

b. [...], que mandan un correo invitando a la gente a ir $\bullet$ se $\bullet \mathbf{n}_{<\text {la gente }>}$ a ver sin solicitarme autorización para hacer una actividad de estas.

Tipo: Acta.

Fuente: Consejo Universitario de la Universidad Estatal a Distancia (2007).

Como lo muestran las oraciones (7)-(12), el plural exclítico resulta de la concordancia con el sujeto de la oración principal de la cual la subordinada infinitiva es dependiente. Como lo señaló Sánchez Corrales (1987), este tipo de plural tiene una función de desambiguación, que en estos ejemplos se trata del sujeto de la infinitiva (PRO), donde se marca claramente su relación con el referente del sujeto en la oración principal. Nosotros consideramos, además, que este tipo de concordancia forma parte de un conjunto de fenómenos que es necesario abordar en su totalidad. En este sentido, la oración en (13) nos permite describir, con mayor detalle, la complejidad del plural exclítico. En dicho ejemplo, el plural exclítico \{-s\} en "los" interactúa también como un plural externo a la secuencia clítica, pero se trata un plural nominal, resultado de un se espurio (Perlmutter, 1970), es decir, de un pronombre plural de objeto indirecto ("les"), realizado como "se" por la aplicación de una regla morfofonológica, donde el plural \{-s\} pierde asidero y se asocia con el único pronombre que tiene una posición de plural vacía ("lo") (13b). Es necesario recordar que el exclítico nominal se produce únicamente con el se espurio (Harris, 1994; 1997). En (13b), los detalles de estas relaciones están indicados explícitamente; el sintagma nominal "Los Nicas" es retomado en las oraciones siguientes, tanto por un pronombre proclítico de objeto directo ("los"), como por el plural exclítico \{-n\} 
en "venirsen". Adicionalmente, el plural exclítico en el pronombre proclítico de objeto directo está referido a dicho sintagma nominal, mientras que el pronombre de objeto directo "lo" propiamente dicho está en relación con el cuantificador universal "todo" que, dicho sea de paso, está en singular, razón por la cual reconocemos el exclítico nominal, tal y como lo representa el análisis de la frase en (13b).

(13) a. Más ayudas para los Nicas. Bueno eso los incentiva a venirsen en grandes cantidades ya que todo se los regalan.

Tipo: Comentario.

Fuente: Angulo (2019).

b. Más ayudas para [los Nicas]. Bueno eso $\operatorname{los}_{<\operatorname{los} \text { Nicas }>}$ incentiva a $\mathrm{PRO}_{<\operatorname{los} \text { Nicas }>}$ venir•se $\bullet n_{<\text {los Nicas }>}$ en grandes cantidades ya que [todo] se $\mathbf{l o}_{<\text {todo }>} \bullet \mathbf{s}_{<\text {los Nicas }>}$ regalan.

Es interesante notar que la elisión de $\{-s\}$ es obligatoria en ciertos contextos del español normativo, como delante del "imperativo (subjuntivo exhortativo)", donde la norma prescriptiva establece "dejémonos" en vez de "dejémosnos" y "pongámoselo" en vez de "pongámosselo" (Real Academia Española y Asociación de Academias de la Lengua Española, 2005).

El plural exclítico no es exclusivo de Costa Rica. Tal y como lo señalan las fuentes bibliográficas, este tipo de plural se extiende a prácticamente todas las zonas de habla hispana. Así, por ejemplo, recopilamos muestras de España (14) y de Colombia (15)-(17).

(14) Aquí vemos a un@s cuantos parásitos que deberían estar trabando en vez de ir a Colón a ponersen cara al Sol.

País: España.

Fuente: Claudia (2019).

(15) Ojo jóvenes programadores de software a ponersen las pilas, me pasaron una información de una fuente.

País: Colombia.

Fuente: Miryam Torres (2018).

(16) Invitan para ponersen al día con el Esquema de Vacunación a niños Menores de 10 años del Municipio

País: Colombia.

Fuente: Alcaldía Municipal de San Andrés en Santander (s. f.).

(17) Insisto! La falta de justicia nos está convirtiendo en asesinos. Es indignate sentirse uno atracado y verlos irsen con nuestras pertenencias sin poder hacer nada lo viví hace unos días atrás.

País: Colombia.

Fuente: Gloria Moreno (2020).

Por otra parte, el plural exclítico verbal no se limita al infinitivo, puesto que también existen formas de imperativo en el que se realiza, como lo ilustran las frases en (18)-(20), tomadas de Harris y Hale (2005). Los ejemplos (18a), (19a) y (20a) siguen la norma estándar panhispánica, mientras que (18b), (18c), (19b) y (20b) muestran realizaciones del plural exclítico, donde $\{-n\}$ entre paréntesis indica una posición facultativa para su realización. Es importante notar que las oraciones (18)-(20) corresponden al ustedeo, en este caso, se trata del uso del pronombre personal de segunda persona plural, "ustedes", cuya morfología coincide 
con la conjugación de la tercera persona del plural ("que ellos vendan", "que ellos le den", "que ellos lo hagan").

(18) a. véndanlo

b. véndanlon.

c. véndalon.

(19) a. denle eso.

b. de(n)len eso.

(20) a. háganlo mejor.

b. hága(n)lon mejor.

En síntesis, en el español de Costa Rica (y en otras variantes panhispánicas) existe una forma de plural incorporado a la posición final de una secuencia enclítica, correspondiente a la morfología verbal, $\{-\mathrm{n}\}$, y no a la morfología nominal, $\{-\mathrm{s}\}$, como es de esperarse, cuyo referente en las subordinadas infinitivas es el sujeto de la oración principal y, en el caso de las oraciones imperativas, el referente de ese plural anafórico es el sujeto del verbo (que tiende a ser desinencial en las formas imperativas). Además, en Costa Rica también encontramos la presencia de un plural anafórico exclítico nominal, producto de una transformación morfofonológica del pronombre de objeto indirecto "les" en "se", conocida como se espurio. El plural exclítico verbal se expresa según la morfología de la tercera persona del plural; por esta razón, aparece en oraciones de tercera persona y también en formas imperativas correspondientes al ustedeo (es decir, en segunda persona). El pronombre que ocupa la posición final enclítica, al cual se incorpora la realización del plural exclítico, debe ser singular, de primera persona -"me"- o de tercera persona -"la", "lo", "le" o incluso "se"-, en acusativo o dativo, para las secuencias verbales.

\section{Hacia una visión de conjunto del plural exclítico}

Aparte de la adición de plural a una secuencia clítica, que denominamos plural exclítico, encontramos la operación contraria, es decir, la elisión del plural en un pronombre clítico (Barbeito, 2017). Concretamente, se trata de la posibilidad de elidir el plural en el pronombre clítico de objeto indirecto, "les", que frecuentemente se realiza como "le" en presencia del objeto indirecto plural que duplica (Bickford, 1985; Pérez, 2000; Baker y Kramer, 2018); es decir, este pronombre siempre concuerda en caso, pero la concordancia de plural no siempre se produce. Los ejemplos en (21) ilustran esta posibilidad, donde (21a) es la forma normativa estándar y (21b) corresponde a la elisión del plural.

(21) a. Les dije eso a mis tíos.

b. Le dije eso a mis tíos.

c. Se los dije a mis tíos.

d. $\mathrm{Se}_{<\text {a mis tíos }>} \bullet \mathrm{lo}_{<\mathrm{eso}>} \bullet \mathrm{s}_{<\text {mis tíos }}$ dije a mis tíos

Es importante llamar la atención sobre esta operación, porque muestra una erosión de la marca pronominal de plural, la cual deja una posición morfológica vacía que, bajo ciertas circunstancias, como en la enclisis, podría ser ocupada por otro morfema de plural, con un referente distinto al del objeto indirecto. Así, queda abierto el espacio para que en (21c) se presenten las condiciones para la realización de un se espurio, "Se $e_{<a}$ mis tíos> $>$ ", con una 
transferencia de plural, analizada en (21d) como " $\mathrm{lo}_{<\mathrm{eso}>} \bullet \mathrm{s}_{<\mathrm{mis}}$ tíos $>$ ", donde la concordancia con el objeto indirecto es de número, a diferencia del se espurio en (21d), donde la concordancia es

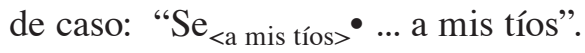

En lo que respecta al plural exclítico del verbo, incorporado a las secuencias enclíticas, tenemos dos tipos. El primero corresponde a las subordinadas infinitivas; el segundo, al imperativo. Este último fue analizado por Harris y Halle (2005), junto con el se espurio, como resultado de una operación denominada Kopy \& VIM, siguiendo la teoría de la Morfología Distribuida, consistente en la ejecución de una serie de acciones postsintácticas de metátesis y reduplicación. Este análisis si bien es cierto da cuenta del imperativo y del se espurio, falla en la explicación de la concordancia infinitiva, dado que el infinitivo, por definición, carece de morfemas que puedan ser duplicados o que puedan ser objeto de la operación de metátesis, razón que nos lleva a considerar la posibilidad de una perspectiva más amplia para el análisis del plural exclítico.

Por otra parte, la realización de un plural exclítico en el imperativo de segunda persona plural correspondiente al pronombre ustedes prueba que no es exclusivo de la tercera persona. Pato y De Benito (2017) presentan ejemplos de "tú", pronombre de la segunda persona del singular, de lo que llaman transposición del clítico, donde secuencias que normativamente deberían ser

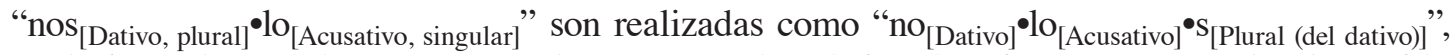
es decir, en las que hay un patrón de un pronombre dativo de primera persona plural seguido de un pronombre acusativo de tercera persona singular sobre el que recae el morfema de plural, donde, por transposición, el plural del pronombre dativo se incorpora al pronombre de objeto directo y obtenemos "no $<$ a nosotros $>$ 'lo $\bullet_{<\text {nosotros }>\text { " en vez de "nos }}$ a nosotros $>$ 'lo". En (22) presentamos una frase adaptada del ejemplo (6a) de Pato y De Benito (2017). No encontramos ninguna muestra de esta transposición en el español de Costa Rica, sin embargo, sigue el mismo esquema de formación del se espurio, con la novedad de que involucra un pronombre clítico de primera persona plural, "nos", en vez de un pronombre clítico de tercera persona plural, "les", transformado en un "se". La posibilidad de incorporación de plurales exclíticos en pronombres de objeto directo está demostrada, puesto que no es exclusividad de los pronombres dativos.

(22) a. ¡Préstanolos un rato!

b. ¡Présta・no $<$ a nosotros $>\bullet^{\bullet} \operatorname{lo}^{\bullet} \mathrm{s}_{<\text {nosotros }>}$ un rato!

Las evidencias presentadas hasta ahora, apuntan a que en el español contamos con dos tipos de plurales exclíticos, uno nominal y uno verbal. El plural exclítico nominal corresponde a lo que parece ser una recuperación de un morfema plural sin asidero luego de la conversión morfofonológica del pronombre "les" en "se" por una operación de se espurio. Además, un patrón similar aparece en otras secuencias de pronombre dativo seguido de un pronombre acusativo singular, ejemplo (22). El plural exclítico verbal, $\{-n\}$, podría ser considerado como una reconfiguración de las secuencias morfológicas del imperativo, de manera que siempre se manifieste el morfema plural en final de palabra, incluso luego de las secuencias enclíticas; sin embargo, la posibilidad de una realización duplicada de dicho morfema, como en (18b), (19b) y (20b), excluye esta hipótesis. La duplicación es una operación común en varias lenguas, por lo que tampoco se puede descartar que opere en el imperativo del español, pero esta hipótesis también está excluida por la realización de una \{-n\} plural en posición postenclítica en el modo infinitivo, por lo tanto carente de formas personales que puedan ser morfológicamente 
duplicadas. La frecuente pérdida del plural en el pronombre de objeto directo "le(s)" en la duplicación clítica deja abierta la posibilidad a otras explicaciones para el plural exclítico, como la existencia de una posición de plural, disociado referencialmente y argumentalmente de los otros elementos en la secuencia clítica, asequible a plurales de sujeto u objeto indirecto.

\section{Conclusiones}

El infinitivo personal o conjugado es un fenómeno presente en varias lenguas, como, por ejemplo, el portugués, el gallego, el sardo y algunos dialectos italianos (Posner, 1996; Carrera y Rodrigues, 2006; Vásquez, 2011), por lo que existe, sin duda, una presión estructural, que data del romance, y que explicaría su persistencia en el español contemporáneo (Sánchez Corrales, 1987).

Ahora bien, sincrónicamente, el infinitivo conjugado llama la atención por su naturaleza incompatible con cualquier norma prescriptiva del español. Tal y como lo hemos presentado en la primera parte de este artículo, ejemplos (7)-(13), el infinitivo personal goza de gran vitalidad en el español de Costa Rica, país en el que también encontramos realizaciones de transferencia de plural, ejemplo (13). El infinitivo personal que analizamos se manifiesta en el pronombre singular enclítico, de acusativo o dativo, en las subordinadas infinitivas, donde el plural al final de palabra corresponde a la morfología verbal, $\{-n\}$, posición que en principio corresponde a un morfema plural nominal, $\{-\mathrm{s}\}$. Analizando los referentes en las frases, encontramos que dicho plural, $\{-n\}$, está asociado con el sujeto de la oración principal y no con los argumentos con los cuales los pronombres clíticos están coindexados. Este tipo de plural, que llamamos exclítico, por no corresponder a ningún referente de la secuencia clítica, también existe en otros países de habla hispana, en (14) brindamos ejemplos de España y, en (15)-(17), de Colombia.

Aunque varios análisis abordan este fenómeno, nosotros abogamos por una visión de conjunto del plural en las secuencias clíticas. Para esto nos apoyamos en varios hechos. En primer lugar, el español presenta una erosión del plural en el pronombre de objeto indirecto, ejemplos (21) y (22), hecho que puede influir también en los pronombres de objeto directo, probablemente debido a motivaciones fonológicas por investigar. Esta erosión deja un espacio disponible para el plural, el cual habría perdido la capacidad de referir al mismo elemento que el clítico que lo hospeda, pero no la posibilidad de contener la carga semántica asociada a un plural. En los ejemplos que presentamos, esa posición está vinculada con el sujeto de la oración principal, en la concordancia de infinitivo, y con el objeto indirecto, en el se espurio, donde el plural se realiza en el pronombre de objeto directo (13). En segundo lugar, proponemos clasificar el plural exclítico del español en dos categorías, una nominal y una verbal. Del primero, en Costa Rica, solo encontramos ejemplos resultado de una operación de se espurio (13), puesto que no hallamos evidencia en el país de secuencias "nos ${ }_{\text {[Dativo, plural] }}{ }^{\circ} \mathrm{l}_{\text {[Acusativo, singular] }}$ " realizadas como "no ${ }_{[\text {Dativo }}{ }^{\bullet} \mathrm{lo}_{[\text {Acusativo }}{ }^{\bullet S}$ [Plural (del dativo)]", como en (22). Los exclíticos verbales en Costa Rica se realizan en el imperativo (conjugado en usted) y en infinitivo subordinado. Si consideramos estos dos fenómenos como operaciones relacionadas, debemos descartar un análisis basado en la reduplicación y la metátesis, ambas imposibles en el infinitivo. Consideramos que ambas versiones del plural exclítico deben ser analizadas por separado.

Queda, sin lugar a dudas, un cierto número de preguntas pendientes, para continuar la investigación. Así, desde el punto de vista teórico, es interesante plantearse el estatus argumental del sujeto y del morfema de plural, el cual, además, presenta una evolución 
semántica que tiende a darle un peso mayor que el de un morfema. En este sentido, un análisis desde el punto de vista de la morfología flexiva se impone, dominio que también debe incluir los pronombres clíticos por su condición ambigua en la frase entre la morfología y la sintaxis. Además, conviene también aumentar el inventario de ejemplos, a fin de establecer si existen realizaciones del plural exclítico en el sujeto de infinitivo (*el salirsen no es bueno), algo que, a priori, no debería ser posible puesto que, según lo que hemos presentado, tienen una función anafórica. Finalmente, también sería interesante abordar, a partir de secuencias como las del ejemplo (22), la presión estructural fonológica en la /s/ final, recordemos que el español normativo prescribe la elisión de la $\{-s\}$ en la conjugación de la primera persona para "dejémonos", por lo que cabría preguntarse si estamos ante fenómenos de origen fonológico, cuya presión estructural provoca una recuperación y reanálisis a nivel morfológico.

\section{Bibliografía}

Angulo, Y. (7 de noviembre de 2019). Presentan proyecto para garantizar derecho al agua potable para habitantes de precarios. elmundo.cr. Recuperado de https://www. elmundo.cr/costa-rica/presentan-proyecto-para-garantizar-derecho-al-agua-potablepara-habitantes-de-precarios/

Alcaldía Municipal de San Andrés en Santander. (s. f.). Invitan para ponersen al día con el Esquema de Vacunación a los niños menores de 10 años del Municipio. Recuperado de http://www.sanandres-santander.gov.co/noticias/invitan-para-ponersen-al-dia-conel-esquema-de-vacunacion

Baker, M. y Kramer, R. (2018). Doubled clitics are pronouns. Natural Language \& Linguistic Theory, 36, 1035-1088.

Barbeito, V.(2017). La ausencia de concordancia entre el pronombre dativo y su referente nominal en esquemas verbales ditransitivos. Pragmalingüística, (25), 50-61. Recuperado de https://revistas.uca.es/index.php/pragma/article/view/2424

Bastida, S. (1975). A propósito de las restricciones de orden en las secuencias de clíticos en español. Revista de Filología Española, 57(1/4), 79-110.

Carrera, M. y Rodrigues, J. (2006). Las equivalencias en español del infinitivo flexionado portugés; una perspectiva contrastiva. Boletin de Lingüística, 18(26). Recuperado de http://ve.scielo.org/scielo.php?script=sci_arttext\&pid=S0798-97092006000200003

Chaves, E. (19 de setiembre de 2018). Con un brillante Keylor Navas, el Real Madrid golea 3-0 a la Roma. El mundo.cr. Recuperado de https://www.elmundo.cr/deportes/con-unbrillante-keylor-navas-el-real-madrid-golea-3-0-a-la-roma/

Claudia. (10 de febrero de 2019). Aquí vemos a un@s cuantos parásitos que deberían estar trabando en vez de ir a Colón a ponersen cara al Sol [Tuit]. Recuperado de https:// twitter.com/Claudia08588610/status/1094701346664730624

Comunicados Vive 506 CR. (8 de setiembre de 2017). Arba San Ramón se proclama campeón del Baloncesto. Vive 506 CR. Recuperado de http://vive.506.cr/deportes/234basket/502-arba-san-ramon-se-proclama-campeon-del-baloncesto

Consejo Municipal de Santa Ana. (29 de julio de 2014). Acta de la sesión ordinaria No. 
221. Recuperado de https://santaana.go.cr/index.php/institucional/el-concejo/actas-deconcejo?task=document.download\&id=766

Consejo Universitario de la Universidad Estatal a Distancia. (14 de agosto de 2007). Acta No. 1879-2007 Extraordinaria. Recuperado de https://www.uned.ac.cr/conuniversitario/ images/actas/2007/1879-2007.pdf

Gloria Moreno. (10 de febrero de 2020). Insisto! La falta de justicia nos está convirtiendo en asesinos. Es indignate sentirse uno atracado y verlos irsen con nuestras pertenencias sin poder hacer nada lo viví hace unos días atrás [Tuit]. Recuperado de https://twitter. com/gloriamoreno248/status/1226855525175918592

Harris, J. (1994). The syntax-phonology mapping in Catalan and Spanish clitics. En A. Camie, H. Harley y T. Bures (Eds.), Papers in phonology and morphology (pp. 321-353). MIT Working Papers in Linguistics 21. Cambridge, Mass.: MIT, Department of Linguistics and Philosophy.

Harris, J. (1997). Morphologie autonome et pronoms clitiques en catalan et en espagnol. En A. Zribi-Hertz (Ed.), Les pronoms : Morphologie, Syntaxe et Typologie (pp. 35-56). Vincennes, Francia: Presses Universitaires.

Harris, J. y Halle, M. (2005). Unexpected Plural Inflections in Spanish: Reduplication and Metathesis. Linguistic Inquiry, 36(2), 195-222. Recuperado de http://www.jstor.org/ stable/4179318

Bickford, J. A. (1985). Spanish clitic doubling and levels of grammatical relations. Lingua, 65(3), 189-211.

Manzini, M. R. y Savoia, L. M. (2011). Mesoclisis in the imperative: Phonology, morphology or syntax? Lingua, 121(6), 1101-1120.

Mare, M. (2018). Una nueva mirada sobre la concordancia inesperada en español. Revista de Filología Española, 28(2). Recuperado de http://revistadefilologiaespañola.revistas. csic.es/index.php/rfe/article/view/1228/1517

Miryam Torres. (7 de mayo de 2018). Ojo jóvenes programadores de software a ponersen las pilas, me pasaron una información de una fuente [Tuit]. Recuperado de https://twitter. com/MiryamTorres7/status/993500764084559872

Mora Hernández, C. (1 de mayo de 2015). Opinión: Doña Epsy, le ofrezco una disculpa y le pido que se ocupe en cosas más serias. Crhoy.com. Recuperado de https://archivo. crhoy.com/opinion-dona-epsy-le-ofrezco-una-disculpa-y-le-pido-que-se-ocupe-encosas-mas-serias/opinion/el-lector-opina/

Pato, E. y de Benito, C. (2017). Tráenolos para comérnolos o la 'transposición' del clítico en español actual. Philologica Jassyensia, 13(1), 121-136. Recuperado de http://www. philologica-jassyensia.ro/upload/XIII_1_Pato.pdf

Pérez, S. (2000). Reduplicación de clíticos en español. En P. Butragueño (Ed.), Estructuras en contexto: Estudios de variación lingüística (pp. 81-102). México, D.F.: El Colegio de México.

Perlmutter, D. (1970). Surface Structure Constraints en Syntax. Linguistic Inquiry, 1(2), 187255. Recuperado de http://www.jstor.org/stable/4177552 
Posner, R. (1996). The Romance languages. Cambridge: Cambridge University Press.

Real Academia Española y Asociación de Academias de la Lengua Española. (2005). Pronombres personales átonos. Diccionario panhispánico de dudas (1 ed.). Recuperado de https://www.rae.es/dpd/pronombres\%20personales\%20átonos

Sala Constitucional de la Corte Suprema de Justicia. (22 de abril de 2009) Sentencia 09-001307-0007-CO [MP. Rosa María Abdelnour Granados]. Recuperado de https:// app.vlex.com/\#vid/499373974

Sánchez Corrales, V. (1987). La ambigüedad como mecanismo del cambio lingüístico: ejemplos del español de Costa Rica.Revista de Filología y Lingüística, 13(1), 163-166. Recuperado de http://revistas.ucr.ac.cr/index.php/filyling/article/download/18787/18922

Vásquez, I. (2011). Reflexiones sobre el infinitivo conjugado portugués desde la perspectiva española. Revista Científica, (5), 9-26. Recuperado de https://dialnet.unirioja.es/ servlet/articulo?codigo $=3696707$ 